\title{
IGS for exploration of Foreign Bodies in the Head and Neck- Lessons to Learn
}

\author{
Ching Yee Chan ${ }^{1}$, Pon Poh Hsu ${ }^{1}$, Alvin Kah Leong Tan ${ }^{1}$ and Jason Siew Yoong Hwang ${ }^{2}$ \\ ${ }^{1}$ Department of Otolaryngology, Changi General Hospital, Singapore \\ ${ }^{2}$ Care Sinus Snoring ENT Centre, Singapore
}

Submission: January 18, 2018; Published: February 15, 2018

*Corresponding author: Ching Yee Chan, Department of Otolaryngology, Changi General Hospital, 2 Simei Street 3 Singapore 529889, Singapore, Tel: 6788 8833, Fax: 6788 0933/+65 98236403, Email: chan.ching.yee@singhealth.com.sg

\section{Abstract}

Objectives: Foreign body ingestion is a common presentation to Otolaryngology departments in Asian countries. Road traffic accidents sometimes result in embedded foreign bodies. Exploration and removal of foreign bodies in the head and neck region can be challenging. Image guided surgery (IGS) has been used successfully in endoscopic sinus surgery. This article explores its role in the removal of foreign bodies in the head and neck.

Methods: Two patients with migrated fish bones underwent open neck exploration with intra-operative imaging and IGS. One patient with an embedded glass fragment in his forehead was explored under local anaesthesia with IGS.

Results: Two of three embedded foreign bodies were successfully located and removed.

Conclusion: The image guidance system assists in localisation of foreign bodies in the head and neck. This allows smaller incisions and shortens operating time. It is more precise when the target foreign body is close to bony structures, which are relatively immobile.

\section{Purpose}

Foreign body ingestion is a common presentation to the Otolaryngology department in Asian countries due to local dietary habits. Ingested foreign bodies that have migrated out of the upper aero-digestive tract require open neck exploration. Patients involved in road traffic accidents often suffer from embedded foreign bodies. Exploration and removal of such foreign bodies is often challenging, as they can be small and located in regions with difficult access and surrounding vital structures. Image guided surgery (IGS) has been used successfully in neurosurgery and endoscopic sinus surgery (ESS) [1]. By registering preoperative or intra-operative images together with patient and instrument trackers, IGS allows correlation of the operative field to the imaging data [2]. This gives the surgeon the position of a selected surgical instrument in relation to the surrounding structures. This allows avoidance of vital structures and also guides the extent of surgical resection. We describe the role and challenges of IGS for the removal of foreign bodies in the head and neck region. Besides using pre-operative imaging we have also integrated the use of intra-operative imaging using a C-arm coupled with IGS. We believe this is the first such description in Otolaryngology literature.

\section{Materials and Methods}

Three patients with head and neck foreign bodies underwent removal using Image Guided Surgery over a six-month period.
Two patients had migrated fish bones and one patient had an embedded foreign body after a road traffic accident (RTA). The first patient developed a foreign body sensation after eating stingray. Lateral neck x-ray showed a density anterior to the $\mathrm{C} 6$ vertebral body. This was confirmed with a computed tomography (CT) scan of the neck. The second patient presented with odynophagia one week after swallowing a foreign body. The lateral neck x-ray showed swelling of the epiglottis and a vague image of a curvilinear foreign body in the base of tongue region. This was further confirmed on the CT scan which shows the foreign body embedded in the right base of tongue.

Both patients with migrated fish bones underwent open neck exploration. Optical tracking with auto-registration using the Medtronic Stealth Station S7 was used together with an intraoperative 3D C-arm (Siemens ARCADIS Orbic 3D). Patients were secured to the operating table to minimise movement and the optical patient reference was fixed to their forehead. This is the master reference which registration is based on. The patient was positioned and the pre-calibrated 3D C-arm and IGS system were brought into the operating theatre. After taking antero-posterior and lateral fluoroscopic images with the $\mathrm{C}$-arm, the foreign body is marked out on the images. This becomes the iso-centre for the $\mathrm{C}$-arm. The $\mathrm{C}$-arm then starts its image acquisition by capturing multiple 2D images surrounding the iso-centre and reconstructs them into 3D volumetric data. 
Following image acquisition, the data is seen on the IGS system screen in a triplanar view (axial, coronal and sagittal planes through the same anatomic point). The foreign body was identified and marked on the images and the IGS was used to localise the foreign body with the navigation pointer.

The third patient had multiple glass fragments embedded in his face following a road traffic accident. Initial wound debridement without IGS removed nine of ten glass fragments seen on the CT scan of the head. Six months later, though there were no visible external signs, he reported intermittent swelling and discharge from the forehead, presumably from the retained glass fragment. Based on preoperative CT scan data, IGS was used to assist in removal of the foreign body under local anaesthesia. The IGS system chosen in this case was the Medtronic Stealth Station S7 with Fusion Electromagnetic ENT application.

\section{Results}

Of the three patients, two had their embedded foreign bodies successfully located and removed with IGS assistance. The first patient with the migrated stingray bone had the foreign body located precisely in the right longus colli muscle adjacent to the vertebral body, near the vertebral artery. The other fish bone embedded in the muscles of the tongue base was not found and postoperative CT scans were negative for foreign body. The third patient had the glass fragment removed under local anaesthesia via a small stab incision directly over it.

\section{Discussion}

Removal of foreign bodies in the head and neck region can be challenging. There have been a handful of reports on the use of IGS to assist in the removal of embedded foreign bodies. These include foreign bodies sustained during trauma [3], surgical procedures and dental work (a broken needle [4], dislocated teeth [5], gunshot pellets [6] and a broken cannula). However, all these reports utilised pre-operative CT images6. We believe our report is the first description of IGS using intra-operative images for the removal of foreign bodies, by coupling a C-arm to an IGS system.

The IGS cases reported by Holmes [4], SieBegger [6] and Eggers [5] used pre-operative CT scans performed with reference markers placed on the patient. These were located on the scalp, skin, skull or within a template for the jaw worn by the patient during the scan. Invasive procedures (such as fixing titanium screws to the skull) were sometimes required to attach the markers. Besides the initial diagnostic imaging, some patients needed repeat scans performed to simulate the surgical position (for example, with the mouth open).

Intra-operative imaging eliminates many of these issues. Imaging is done in the exact surgical position, minimizing foreign body and soft tissue movement, which is inevitable during the interval between pre-operative scans and the actual time of surgery. This gives maximum accuracy and would additionally avoid the need for pre-operative markers, especially titanium screws.

The optical IGS system has an infrared camera system that tracks the spatial position and orientation of markers, which reflect infrared light emitted from the camera itself. Such markers are located on the optical patient reference, the wireless C-arm reference tracker and the navigation instruments. Therefore, the location of the $\mathrm{C}$-arm detector is tracked by the IGS during image acquisition and the relation between the coordinates of the $\mathrm{C}$-arm and image coordinates in space together with the 3D dataset are automatically transferred to the IGS system. This provides direct coupling with the navigation instruments. As such, manual navigation registration is not required and the accuracy achieved is $1.5 \mathrm{~mm}$ and below.

IGS allows intra-operative correlation of the surgical field and instrument position. The probe is directed towards the foreign body and the surrounding tissue is dissected until the foreign body is located7. The potential benefits would include shorter operating time, decreased injury to surrounding structures, minimally invasive access and faster patient recovery $[6,7]$. Though there are concerns with cost and availability, the use of IGS should be considered for all embedded foreign bodies in the head and neck as there is minimal morbidity with large potential benefit. We suggest the use of IGS in the following clinical scenarios.

\section{A. Small foreign bodies.}

B. Proximity to vital structures with potentially high risk of morbidity [5].

C. Presence of multiple foreign bodies.

D. Failure of previous removal attempt [5].

Lessons learnt when using IGS in the search for embedded foreign bodies include:

a. IGS is less useful when the foreign body is embedded in a large soft tissue mass. Its accuracy is higher when the target foreign body is close to bony structures, which are relatively immobile [5]. This is exemplified in our second patient as the fish bone was embedded in the muscle mass of the tongue base. Patient one and three had foreign bodies close to bony structures resulting in rapid identification of the foreign body.

b. Pre-operative scans can be unreliable due to migration of the foreign body during the interval between the preoperative scan to the time of surgery [4]. This is demonstrated in patient one, when the foreign body moved laterally in the 18 hours between the times of first CT scan to the time of the intra-operative scan using the $\mathrm{C}$-arm.

c. Changes in the surgical site performed intra-operatively are not reflected on the images obtained using the $\mathrm{C}$-arm [8]. Though the $\mathrm{C}$-arm images are taken with the patient 
on the operating table, minimising any possible error, it is inevitable that the surgery itself results in movement of the foreign body, leading to inaccuracies.

\section{Conclusion}

For optimal use of IGS, we recommend

i. Perform C-arm imaging with the patient in the appropriate surgical position just prior to exploration.

ii. Meticulous dissection to decrease intra-operative movement of the foreign body.

iii. Consider re-imaging with the $\mathrm{C}$-arm when dissection reaches the depth of the foreign body, especially when the foreign body cannot be located as there may have been intraoperative migration.

iv. IGS does not negate the need for anatomical knowledge. The close proximity of vitals structures makes neck exploration a potential minefield and should be led by an experienced surgeon.

\section{References}

1. Freysinger W, Gunkel AR, Thumfart WF (1997) Image-guided endoscopic ENT surgery. Eur Arch Otorhinolaryngol 254: 343-346.

2. Peters TM (2006) Image-guidance for surgical procedures. Phys Med Biol 51(14): R505-R540.

3. Vairavan N, Tajunisah I, Subrayan V, Waran V (2009) Minimally Invasive Image-Guided Removal of Retrobulbar Intraconal Foreign Body Orbit 28(6): 442-443.

4. Holmes PJ, Miller JR, Gutta R, Louis PJ (2005) Intraoperative imaging techniques: A guide to retrieval of foreign bodies. Oral Surg Oral Med Oral Pathol Oral Radiol Endod 100(5): 614-618.

5. Eggers G, Haag C, Hassfeld S (2005) Image-guided remvoval of foreign bodies. Br J Oral Maxillofac Surg 43(5): 404-409.

6. SieBegger M, Mischkowski RA, Schneider BT, Krug B, Klesper B, et al. (2001) Image guided surgical navigation for removal of foreign bodies in the head and neck. J Craniomaxillofac Surg 29(6): 321-325.

7. Halling F, Merten HA, Dieckmann G, Luhr HG (1991) Stereotatic removal of foreign bodies in the maxillofacial area. Dentomaxillofac Radiol 20(2): 100-104.

8. Peters TM (2000) Image-guided surgery: from X-rays to virtual reality. Comput Methods Biomech Biomed Engin 4(1): 27-57.

\section{Your next submission with Juniper Publishers will reach you the below assets}

- Quality Editorial service

- Swift Peer Review

- Reprints availability

- E-prints Service

- Manuscript Podcast for convenient understanding

- Global attainment for your research

- Manuscript accessibility in different formats

( Pdf, E-pub, Full Text, Audio)

- Unceasing customer service

Track the below URL for one-step submission https://juniperpublishers.com/online-submission.php 